\title{
Cuestiones de aula. Gestión curricular
}

\author{
Classroom issues. Curriculum management
}

Recibido junio 2020

Arbitrado julio 2020

Publicado septiembre 2020
Eloy Omar Siñani Alaro

doneomarsa@gmail.com

Código ORCID: 0000-0002-2810-8465

U.E “Álvaro García Linera” - Vinto, Bolivia

\section{Resumen}

El liderazgo educativo es la influencia interpersonal ejercida en un determinado espacio de interacción social, dirigida a través del proceso de comunicación humana a la consecución de uno o diversos objetivos específicos dentro de la institución educativa. La presente investigación se realiza en el aula del 3er año de escolaridad de la Unidad Educativa Álvaro García Linera, Distrito Educativo Vinto, para conocer la percepción de los estudiantes sobre el liderazgo del director, ya que esto repercute en la gestión educativa. Este trabajo buscó responder a las siguientes interrogantes: ¿Cómo es el liderazgo del director desde la percepción de los estudiantes? ¿Cuáles son los rasgos del liderazgo del director? La propuesta que se presenta es un aporte teóricopráctico que posibilitará contribuir en alguna medida con esta problemática, a través de la capacitación que se desarrolla en cuatro talleres sobre: relaciones humanas, comunicación, liderazgo educativo, cultura organizacional y clima laboral.

\footnotetext{
Abstract

Educational leadership is the interpersonal influence exerted in a certain space of social interaction, directed through the process of human communication to the achievement of one or several specific objectives within the educational institution. This research is carried out in the classroom of the 3rd year of schooling of the Álvaro García Linera Educational Unit, Vinto Educational District, to know the perception of students about the leadership of the director, since this affects educational management. This work sought to answer the following questions: How is the leadership of the director from the perception of the students? What are the manager's leadership traits? The proposal that is presented is a theoretical-practical contribution that will make it possible to contribute to some extent with this problem, through the training that is developed in four workshops on: human relations, communication, educational leadership, organizational culture and work environment.
}

\author{
Palabras clave: \\ Liderazgo \\ educativo; \\ dirección; docencia; \\ comunicación; aula
}

\author{
Keywords: \\ Educational \\ leadership; \\ direction; teaching; \\ communication; \\ classroom
}




\section{INTRODUCCIÓN}

$\mathrm{D}$ esde el punto de vista social - educativo en las instituciones escolares se manifiestan distintas maneras de participación, organización, dirección e integración y, por supuesto, diferentes modelos de liderazgo. Ser directivo no es tarea fácil, es asumir grandes responsabilidades encaminadas en la consecución de los objetivos organizacionales, y que deberían aunar sus esfuerzos tendientes hacia una gestión educativa de calidad, en mejora de la realidad en que se vive.

Asimismo, Carrera (2002) realizó un estudio descriptivo sobre las tendencias de liderazgo en el docente venezolano de la I etapa de Educación Básica. La investigación pretendió determinar las características más resaltantes del liderazgo de los docentes de la I etapa de Educación Básica. Para ello se utilizó como fundamento el modelo del instrumento de Bass y Avolio que permite discriminar entre tres constructos: líder transformacional, líder transaccional y el liberal o laisser faire. El trabajo se orienta, según sus objetivos, hacia un estudio de casos. La población estuvo constituida por 37 maestras y 120 alumnos de tercer grado de la Unidad escolar Colegio "María Inmaculada", institución incorporada a los Proyectos Pedagógicos propuestos en el Currículo Básico Nacional de 1998.

Desde el punto de vista ideal, el buen desempeño de un líder directivo, conlleva a que los integrantes de su equipo trabajen y se desarrollen plenamente, en un ambiente de cordialidad. Esta investigación es relevante porque sus resultados permiten conocer cuál es la percepción que tienen las estudiantes del nivel de liderazgo que predomina en el director de la Unidad Educativa Álvaro García Linera y como éste se relaciona con el desempeño laboral de las docentes.

Adicionalmente, es importante ya que los resultados obtenidos servirán de base para aportar algunas alternativas de solución a situaciones problemáticas que se vienen presentando en las instituciones educativas del Departamento en favor de la mejora de la calidad de la gestión directiva y del desempeño laboral docente. 


\section{Marco Teórico}

\section{Definición conceptual}

Percepción del liderazgo del director. Según Chiavenato (1989) el liderazgo se define como "la influencia interpersonal ejercida en una situación, dirigida a través del proceso de comunicación humana a la consecución de uno o diversos objetivos específicos". (p.19).

Desempeño laboral del docente.

Según Chiroque (2006) se define como: un conjunto de acciones o comportamientos observados en los docentes que conllevan al logro de los objetivos de la organización, y que pueden ser medidos en términos de las competencias de cada individuo y su nivel de contribución a la institución (p. 33).

\section{Definición operacional}

Liderazgo del director. El liderazgo directivo evaluado a partir de la consulta al personal relacionado con la gestión. El instrumento a utilizar en esta evaluación consta de 3 preguntas que se realizó inicialmente, con escalas de 1 a 5 , indicando como respuesta nunca (1), casi nunca (2), a veces (3), con frecuencia (4) y siempre (5). Posteriormente en diálogos en profundidad y grupos de discusión se amplió las percepciones de los estudiantes de 3er año de escolaridad nivel primario, dado que se pretendía ampliar la verbalización de sus pensamientos.

Desempeño escolar. Esta variable presenta tres dimensiones en estudio: motivación para el trabajo, responsabilidad y participación. El instrumento a utilizar es un cuestionario del desempeño escolar laboral de los docentes que consta de 30 ítems, con escalas de 1 a 5 , indicando como respuesta nunca (1), casi nunca (2), a veces (3), con frecuencia (4) y siempre

\section{METODOLOGÍA}

\section{Delimitación de la experiencia}

Experiencia del desarrollo curricular y percepción de liderazgo de los estudiantes de la Unidad Educativa Álvaro García Linera Distrito Vinto - Cochabamba".

La hipótesis fue: Si el liderazgo eficaz garantiza una eficiente gestión educativa entonces, el escaso liderazgo del Director estaría 
influyendo en la percepción que tienen los estudiantes de la Unidad Educativa Álvaro García Linera del distrito educativo Vinto. La variable independiente: Escaso liderazgo del Director. La variable dependiente es: Limitada percepción de los estudiantes.

\section{Objetivos de la Sistematización}

Estos objetivos responderán el para qué hemos sistematizado, cuál los objetivos del mismo.

\section{Objetivo Teórico}

Reflexionar y valorar críticamente los aspectos más relevantes del liderazgo del Director de la Unidad Educativa Álvaro García Linera.

\section{Objetivo Práctico}

Contribuir criterios de análisis y reflexión a partir de las percepciones de los estudiantes de la Unidad Educativa Álvaro García Linera.

\section{Técnicas e instrumentos}

A continuación realizaremos una descripción breve de las técnicas o instrumentos utilizados:

Diálogos en profundidad, porque permiten tener contacto directo con los estudiantes que participaron de manera directa

Grupos de discusión, para recoger de manera dialogada las distintas interpretaciones de los estudiantes

Cuestionario, un mecanismo de relevamiento de información

Estos instrumentos y técnicas contemplaron la disponibilidad de tiempo.

Para realizar el trabajo del relato individual fue necesario realizar una línea de tiempo que nos sirviera para enmarcarnos en ese espacio, fueron a partir de análisis y reflexiones grupales que identificamos hitos relevantes de la gestión curricular como docente de aula. 


\section{VIVENCIA PEDAGÓGICA}

\section{Relato individual}

Soy el Lic. Eloy Omar Siñani Alaro, docente de aula de la Unidad Educativa Álvaro García Linera, dependiente de la Dirección Distrital de Educación Vinto, anteriormente estuve en la localidad de Shinahota en la Unidad Educativa Demetrio Canelas.

Lo que me motivó para realizar este trabajo de investigación posterior al Diplomado fue precisamente la especialidad con la que dio a conocer el Ministerio de Educación "Formación para la Transformación de la Gestión Educativa en el Modelo Educativo Sociocomunitario Productivo", precisamente por el denominativo llegué a deducir que si bien para las maestras y maestros se realizó los cursos de licenciatura en el Programa de Formación Complementaria para Maestras y Maestros con el fin de que el/la docente adquiera las herramientas, pautas, didácticas para la praxis de la implementación del Modelo Educativo Socio Comunitario Productivo en donde se interiorizaron para la concretización misma de lo mencionado, a partir de ello tenía esa misma visión en la que con este diplomado todos los administrativos íbamos a adquirir todos los insumos, herramientas, instrumentos, estrategias, etc. para efectivizar desde la parte administrativa el Modelo Educativo Socio Comunitario Productivo, si bien es la cuarta vez desde una orientación de investigación acción, personalmente realicé un autoanálisis reflexivo donde puedo indicar que aún tengo limitaciones para dirigir con eficacia y eficiencia óptima a partir del enfoque que nos plantea el MESCP.

En ese contexto, el intento de romper ese esquema pasiva y que la revolución democrática y cultural que plantea la Ley 070, me permite implementar y fortalecer la educación descolonizadora, comunitaria, productiva, intra-intercultural, plurilingüe, científica técnica-tecnológica y artística, como un hecho social que ya se vive.

Ya que el hombre y/o mujer es un ser pleno e integral con una educación en la vida y para la vida, a partir de la práctica de los valores comunitarios de reciprocidad, complementariedad, solidaridad, equilibrio, respeto y equidad social, cultural, complementario con la naturaleza el hombre y el cosmos.

En consecuencia, la primera y más importante prioridad de mi Gestión fue el de establecer un clima institucional de confianza con los diferentes sujetos; docentes, estudiantes, consejo educativo 
comunitario, las autoridades locales y del municipio. Para que de esta manera se promueva y/o se fortalezca la coordinación, las relaciones interpersonales, las relaciones interinstitucionales, la buena convivencia entre pares y por consiguiente descubrir habilidades, destrezas en sus desempeños de las maestras para emprender nuevos retos con el modelo educativo socio-comunitario productivo.

Ahora bien, lo que más destaco y son aspectos más resaltantes para mi persona como gestor educativo de los cinco módulos del diplomado son;

Del primer módulo, "Política, Realidad y el Rol Transformador del Gestor Educativo en el Modelo Educativo Sociocomunitario Productivo, en el que hace referencia que el Gestor Educativo debe leer su realidad, investigar su realidad, para luego posicionarse frente a ella y consecuentemente comprometerse con la transformación de la misma, para ello nos plantea una Gestión educativa integral en la práctica del gestor del MESCP enfatizando cuatro elementos para describir la realidad: el aspecto político ético, convivencia, técnico pedagógico y técnico administrativo.

A partir de ello, nos plantea una estrategia o pauta frente a una situación problemática suscitada en las unidades educativas, anteriormente los administradores posiblemente desestimaban en dar solución o manifestaban una actitud pasiva frente a problemáticas y dejaban pasar, sin embargo con las pautas y/o herramientas que nos plantea el MESCP por medio del diplomado nos sugiere un cambio de actitud, de búsqueda de solución frente a problemáticas y/o necesidades que posiblemente han de surgir en la unidad educativa, por lo que rescato el cuadro siguiente donde se establecen cuatro elementos con su respectivo análisis, posibles soluciones y fuentes de respaldo. 
Cuadro 1.Visión integral de la práctica del Gestor en el Sistema Educativo Plurinacional

\begin{tabular}{|c|c|c|c|}
\hline Aspectos/elementos & $\begin{array}{c}\text { Análisis } \\
\text { integral del } \\
\text { problema }\end{array}$ & $\begin{array}{l}\text { Acciones } \\
\text { de } \\
\text { solución }\end{array}$ & $\begin{array}{c}\text { Fuentes de } \\
\text { respaldo } \\
\text { (especificar } \\
\text { normativa } \\
\text { vigente } \\
\text { documentos } \\
\text { curriculares) }\end{array}$ \\
\hline $\begin{array}{l}\text { - Técnico pedagógico } \\
\text { - Administrativo } \\
\text { - Convivencia/relaciones } \\
\text { - Político-ético(sentido de } \\
\text { las acciones educativas) }\end{array}$ & & & \\
\hline
\end{tabular}

Este cuadro me facilitó mi labor como gestor para la resolución de conflictos, ya que en mi unidad educativa implemente dos aspectos o elementos que en su momento se presentó como ser: técnico pedagógico, en el que se observó en las maestras ciertas limitaciones en la elaboración de sus documentos e instrumentos de trabajo las mismas que en algunos casos no se llevaban a efecto, por ejemplo en sus planificaciones se observaba de que no existía la integración, la incoherencia de las orientaciones metodológicas y los criterios de evaluación que se notaba la inconsistencia con respecto al objetivo holístico. De esta manera se tomó algunas acciones a través de actividades de reforzamiento para la apropiación y/o efectivización del MESCP para minimizar las falencias.

El otro elemento que también desarrolle como producto del curso del Diplomado, fue cuando se manifestaron algunas interacciones de discrepancias entre maestras, el de convivencia/relaciones, en aquel entonces desarrollamos un taller de reflexión en cuanto a la importancia de la búsqueda del vivir bien, tomando como premisa el efecto que produce ser MAESTRO/MAESTRA para con los sujetos de la unidad educativa, también se aprovechó en socializar y/o retroalimentar en cuanto a normativas vigentes tales como, el manual de funcionamiento para Unidades Educativas, R.S.212414, etc.

Así mismo, también hago mención de experiencias en relación a lo aprendido de este diplomado en el que se hacía referencia al dialogo escucha, dialogo dialogal, trabajo en redes, buena 
convivencia dentro y fuera de la Unidad Educativa, etc. por lo que el cambio de actitud que fortalecí fue la búsqueda de estrategias para reducir la mayor cantidad de estudiantes que constantemente llegaban atrasados, aquellos estudiantes que muy a pesar de vivir cerca a la unidad educativa llegaban tarde o si bien llegaban puntual tenían el hábito de retornar a su casa por no traer sus materiales escolares y esto mostraba una ausencia de seriedad en la unidad educativa como también perjudicaba el desarrollo normal de las actividades en aula.

A partir de ello realice el dialogo escucha, inicie a diagnosticar el porqué de su retraso de pronto aludían indicando que las madres o padres les asignaba tareas antes de venir a la escuela y que por eso era su retraso otros mencionaban que estaban todavía haciendo sus deberes, en fin cada estudiante con cierta excusa por su retraso, bueno viendo esa situación como estrategia les apunté en un acta comprometiéndose en llegar puntual que en otra si se observase la misma situación se llamaría a la madre o padre de familia, aun así seguían con ese proceder, a los que incurrían dos o tres veces se les enviaba un comunicado indicando que debe mandar más antes para que llegue en hora el o la estudiante, ni aun así no existía cambio de actitud con los estudiantes, por lo que volví a escatimar esfuerzos socializando el reglamento interno muy a pesar de que a inicio de la gestión escolar se les entrego este documento a todas las madres y padres de familia.

En esa razón les pedí a las maestras que me informasen y me dieran parte cuando tendrían reuniones de curso para concientizar a las madres y padres de familia al respecto, es así que volví a socializar en cuanto al reglamento interno así mismo sus alcances y la importancia que tiene este documento para fortalecer su aprendizaje y formación integral de las niñas y niños, en su mayoría de los padres de familia estaban de acuerdo en la aplicación misma de lo mencionado a partir de ello todos firmaron un acta en donde están de acuerdo de su aplicación de reglamento.

Ahora bien, como dice el dicho hecha la ley hecha la trampa, empecé a efectivizar la normativa de la unidad educativa claro está amparado a normas que rigen el funcionamiento de las unidades educativas, de pronto cuando se realizó este reglamento interno y se aprobó en una asamblea de padres y madres de familia en aquel 
entonces no le dieron importancia y tampoco se procedió a aplicar de ahí que se presentó y se presentan conflictos en la labor de un administrativo, sin embargo los insumos o herramientas que nos facilitaron en el curso del diplomado me ayudo bastante como gestor.

Si bien la normativa indica que cuando llegasen atrasados las y los estudiantes se retiene hasta el primer periodo, a partir de ello lo que se hizo fue buscar alternativas, por ejemplo de aquellos que han llegado tarde se les asigno actividades y/o tareas en la que aquellos mientras quedaban retenidos hasta la culminación del primer periodo reforzaban su aprendizaje con actividades, así mismo se les envió comunicados a la madre o padre de familia para que conozcan que su hija o hijo tuvo cierta amonestación y envíen más temprano a la escuela el control de aquello lo realizaba las maestras con sus respectivas firmas de la madres y/o padre en el comunicado que anteriormente se les envió, como efecto hoy se observa casi cero de estudiantes con retraso a la unidad educativa, digo casi porque en algún momento si se presenta de aquellos estudiantes que viven muy distante a la unidad educativa y claro se entiende de estos estudiantes porque hacen mérito en llegar.

En cuanto a la realización de actividades programadas de la gestión con el plantel docente, tenía la costumbre de llevar a efecto de la siguiente manera: socializaba la actividad en una reunión y posteriormente a ello publicaba mediante instructivo para su ejecución y/o implementación bueno esto conllevaba a que las maestras y maestros realicen estas actividades de manera obligada sin compromiso y de pronto las acciones que se realizaban era tan solo por realizar sin la apropiación misma de llevar adelante tal actividad de la mejor manera como resultado que hay veces no salía bien la actividad.

Entonces con este nuevo enfoque que nos plantea los módulos de estudio del diplomado en el que enfatiza el dialogo dialogal, la reciprocidad, el de promover la participación, el de inclusión a los demás sujetos de la comunidad educativa, las relaciones interpersonales, etc. Se hizo el cambio de actitud como gestor, a partir de la coordinación, fortaleciendo las ideas para realizar actividades educativas en reuniones en donde las maestras dan a conocer sus ideas para consolidad actividades consensuadas y donde todos se sienten comprometidos e identificados para su realización. 
Claro que a inicio se observaba grupos de maestras en las que persistían en plasmar y aferrarse a sus sugerencias, era bastante dificultoso consensuar armonizar pero en fin mediante las respectivas reflexiones de la labor del maestro, gradualmente se apaciguó las asperezas entre pares.

Con respecto a la coordinación como gestor y el Consejo Educativo, en la anterior gestión era una de las fortalezas y oportunidades que tuve como gestor ya que existía bastante entendimiento y combinación de ideas para realizar actividades, asimismo el de proyectarnos con algunos requerimientos en bien de las niñas y niños, muchos de los requerimientos obtenidos con resultados óptimos fue precisamente al engranaje de coordinación que propicio.

Sin embargo, en esta gestión se realizó el cambio de los consejos educativos porque ya se había terminado su gestión de los consejos educativos de ahí que desde la distrital comunicaron la nueva conformación de Consejos Educativos.

Es así que realizaron el cambio del consejo educativo en una asamblea de madres y padres de familia en el mes de febrero, se renovaron todas las carteras, como resultado fue elegida Dña. Rosa Barquera que a la vez mencionaba que en una anterior oportunidad participo como miembro de la junta escolar.

A principio como gestor tenía la seguridad de que iban a ratificarle al anterior junta escolar por todas las acciones que se realizó en bien de la unidad educativa pero no fue así, una vez conociendo el cambio del consejo educativo tenía la seguridad que con el nuevo consejo educativo se iba a dar esa coordinación, sin embargo no se mostró así ya que la nueva representante dl consejo empezó a tomar algunas atribuciones que no le correspondían, el de observar el trabajo de las maestras, llamar la atención a la portería, el de su observancia en cuanto a la hora de ingreso y salida de aula de las maestras, de ahí que tome la decisión en reunirlos con todos los sujetos de la unidad educativa; convoque al pleno del consejo educativo, las maestras, los representantes de padres y madres de familia por cada curso, en donde a través de ese espacio se socializó las normativas en cuanto al funcionamiento de las unidades educativas y como quien dice nos enmarcamos en nuestras funciones 
pero eso si con la premisa de coordinación para las diferentes actividades en beneficio de las niñas y niños de nuestra unidad educativa.

Por otra parte como gestor educativo observe que, existen algunas maestras en su persistencia con una didáctica y praxis de enseñanza conductista y que están enmarcados con esta práctica que les resulta menor esfuerzo y menor tiempo de dedicación, posiblemente sean maestras que aún se encuentran encuadrados en lo rutinario, con una actitud pasiva no innovador, donde no escatiman esfuerzo tiempo y dedicación para buscar estrategias e innovaciones en las que puedan facilitar el aprendizaje de los contenidos en los estudiantes, a pesar de que la mayoría de los maestros es decir el 100\% realizaron los cursos del Programa de Formación Complementaria para Maestras y Maestros en dónde se formaron para fortalecer el proceso de implementación del Modelo SocioComunitario Productivo.

Al respecto, como gestor se les hizo la respectiva reflexión a las maestras a partir del dialogo dialogal, donde se promovió la confianza recíproca en cuanto a sus fortalezas y debilidades sobre la dinámica que ejercen dentro del aula de cada maestra, esta dinámica presupone según el modulo tres del Diplomado ..."la caza de informaciones realizada por una razón instrumental”...donde el único propósito de este espacio fue el de que cada maestra cambie de actitud para la implementación de los instrumentos y elementos que conlleva la Ley 070 para su aplicación.

Sin embargo, para que se totalice la implementación del MESCP de parte de los maestros debiera de existir una normativa en la que exija la aplicación de lo mencionado, tal vez así despertarían en poner el esfuerzo y dedicación con todo los elementos que concierne el Modelo Educativo Socio Comunitario Productivo.

También como gestor se promovió la integración de todos los miembros o sujetos de la comunidad educativa, a través de la implementación del Proyecto Socio Productivo de la unidad educativa con la temática "fortalecer hábitos alimenticios en la comunidad educativa para vivir bien", a partir de ello para su elaboración de la misma, los sujetos de la unidad educativa interactuamos desde la 
identificación de la problemática y las acciones planteadas para responder o solucionar lo identificado en el diagnóstico, diagnostico que se realizó mediante la aplicación de instrumentos de recolección de datos.

A partir del diagnóstico, se pudo observar que nuestros estudiantes no conocían el porcentaje de nutrientes que necesitan nuestro cuerpo para tener una vida sana y por lo tanto después de analizar y reflexionar sobre los datos obtenidos de nuestra encuesta, se ve la necesidad de concientizar a nuestros estudiantes sobre los nutrientes equilibrados que tenemos que consumir las personas.

Además que con este tema se puede articular con los cuatro campos de saberes y conocimientos, como también se recupera las buenas costumbres alimentarias de nuestros abuelos y abuelas que en su momento se consumían de una forma natural, producto de ellos nuestros abuelitos y abuelitas llevaron una vida sin enfermedades crónicas por así decirlo.

De acuerdo a una entrevista realizada a los especialistas (profesionales nutricionistas) a través de un cuestionario elaborado para conocer en cuanto a los alimentos que son imprescindibles para la vida y que se suministran al organismo los nutrientes y energía necesarias para mantenerse saludable. Donde indican que para mantener la salud debemos conocer nuestras necesidades nutricionales así como la composición de los alimentos. De este modo podremos analizar si nuestra forma de alimentarnos es la correcta para modificar nuestra dieta si fuera necesario.

Después de aplicar el cuestionario de diagnóstico, a nuestros estudiantes, se concluyó que en una gran mayoría consumen mucha cantidad de grasas, como por ejemplo: en carnes, embutidos, fritos; comida chatarra, como: Enlatados, salteñas, asaditos, hamburguesas, etc.

También se evidenció que nuestros educandos y la comunidad educativa en su conjunto consumen muy poco las frutas, verduras y agua, que según el cuadro son parte de su dieta diaria con poca preferencia los derivados de la leche, dulces, carnes, arroz, entre otras, a pesar de ser una comunidad agrícola.

Todo esto se observó antes de la implementación del plan de acción del Proyecto Socio Productivo "fortalecer hábitos alimenticios en la comunidad educativa para vivir bien", posterior a ello se percibe 
que mejoraron sustancialmente el número y el porcentaje de los alimentos que se deben consumir sanamente.

Para recolectar datos precisos del efecto de la experiencia transformadora en nuestros estudiantes, se utilizó la técnica de cuestionario de diagnóstico, un cuestionario final para conocer y para reconocer el impacto en nuestra comunidad se utilizó la entrevista por medio de un cuestionario además de la observación por quienes aplicaron en este caso los estudiantes y como perciben el liderazgo del Director de la unidad educativa Álvaro García Linera.

No sin antes que en el proceso de la elaboración del PSP con la temática de "fortalecer hábitos alimenticios en la comunidad educativa para vivir bien" con todos sus elementos, se estructuró el plan de acción por bimestres, donde cada bimestre estaba definido mediante actividades bien determinadas tal es de que:

En el primer Bimestre se planteó la actividad de "Fomentar el consumo de Cereales en el desayuno", donde en cada curso o grado con la participación de los sujetos desarrollaron, promovieron y fortalecieron el consumo de un desayuno nutritivo ya que se observaba en nuestras niñas y niños en horas de clase un rápido desgaste de concentración al momento de atender las explicaciones de los contenidos que desarrollaba la maestra.

En esa razón cada curso aprendió a preparar un desayuno nutritivo en colaboración de sus madres de familia, como también desarrollaron contenidos con referente a cereales y que al culminar el bimestre se realizó una feria expositiva para degustar la variedad de desayunos nutritivos.

Para el Segundo Bimestre la acción del plan del PSP fue el de "Promover el deleite de las ensaladas de Verduras y Frutas", de la misma manera se trabajó comunitariamente entre todos los sujetos, pero durante el consumo de las ensaladas se percibió que las niñas y niños no estaban acostumbrados en consumir ensalada de verduras es más desconocían el nombre de algunas verduras, cada maestra integro los contenidos del currículo base con el plan de acción planteado para el segundo bimestre, como producto se realizó una mini-feria de ensaladas de verduras y frutas.

Para el Tercer Bimestre se planificó el "Cambiar hábitos consumiendo Jugos y Refrescos". 
Por último, el Cuarto Bimestre se desarrollará la "Revalorización de los Platos Ancestrales Nutritivos", hago notar que estos dos últimos bimestres se encuentran en proceso de implementación.

De esta manera todos los sujetos que conllevan la unidad educativa como ser las madres y padres de familia, consejos educativo, maestras y estudiantes trabajaron en forma armónica claro que en algún momento se suscitó pequeñas discrepancias que en el momento como gestor educativo se intervino de acuerdo al elemento de "Convivencia" que nos plantea el diplomado para la resolución de conflictos, a través del dialogo, escucha y concertación.

Anteriormente, mi trabajo como administrativo de la unidad educativa, de pronto ha sido respondiendo con una mirada personal de acuerdo a mis criterios, sin embargo con este nuevo enfoque me permitió ver que la gestión sea vista como algo compartido y construido, asimismo lograr transformar la practica educativa al interior del espacio de la unidad educativa.

\section{Discusión}

En esta investigación acción se estudió la relación que existe entre la variable liderazgo directivo y el desarrollo curricular, desde la percepción de los estudiantes del 3er año de escolaridad nivel primaria Unidad Educativa Álvaro García Linera, las cuales en su condición de niños que por lo general no se considera sus percepciones y pensamientos, no solo se dedican a ser estudiantes el cambio de rol como hijos, como hermanos frente a sus compañeros de clases. Allí la riqueza de los resultados de la misma enfocadas al liderazgo del director de la Unidad Educativa, y que pocas veces por lo general interactúa y/o perciben a un déspota, autoritario, con la imagen desprestigiada que tienen los docentes en general, tienen que mostrar polifuncionalidad para cumplir con su labor educativa bajo el monitoreo y control del director, esto fue lo que motivó la realización del presente estudio para indagar sobre la influencia del director (líder) en el desarrollo curricular de los estudiantes.

Al 100\% de los estudiantes les agrada que el Director los reciba en la puerta, solo el $2 \%$ demuestra indiferencia a la tristeza que les provoca cuando no se encuentra el Director en la puerta. 
Al 100\% les pone en una condición de mucha alegría cuando el Director ingresa al aula para revisar los cuadernos porque siempre los felicita a cada uno.

Al 92\% les agrada que el propio director les explique las actividades de la semana, más aún cuando son estos de extensión en los barrios circundantes de la Unidad Educativa

Los resultados obtenidos y analizados estadísticamente han conducido a establecer que existe relación significativa moderada entre la percepción de liderazgo directivo y desempeño de los estudiantes en el desarrollo curricular, además se perciben en los diálogos en profundidad que influye el liderazgo directivo en la responsabilidad de los estudiantes del 3er año de escolaridad nivel primaria Unida Educativa Álvaro García Linera. Lo afirmado anteriormente corrobora el planteamiento de Thieme (2005) quien buscó la relación existente entre liderazgo y desempeño docente. Ellos obtuvieron resultados positivos en cuanto a correlación y la predominancia de la dimensión motivación en relación con el liderazgo directivo, similares los resultados de nuestra investigación.

Finalmente se pone en evidencia que la imagen de autoridad del Director cuando esta se acerca al menos a tener intercambios de opiniones con los estudiantes, cuando se abren espacios de interrelaciones motivacionales hacia los estudiantes puede incidir favorablemente en el buen desarrollo curricular de los estudiantes, aspecto que sin duda tiene relación con el liderazgo directivo con calidad de gestión. Este último será otra iniciativa que se intentará poner en descubierto en la siguiente investigación acción.

\section{CONCLUSIONES}

$\mathrm{N}$ o existe una relación directa significativa entre la percepción del liderazgo del director y el desempeño escolar de los estudiantes, aunque si manifiestan que el estado de ánimo con el que les recibe en la puerta el Director a los estudiantes del 3er año de escolaridad predispone una actitud de aprendizaje en la Unidad Educativa Álvaro García Linera, Distrito Educativo Vinto.

Existe una relación moderadamente significativa entre la percepción del liderazgo del director y la dimensión motivación para 
el desarrollo curricular desde la perspectiva de los estudiantes especialmente al ingreso a clases

Existe una relación significativa moderada entre la percepción liderazgo del director y la dimensión participación de actividades convocadas por el Director desde la perspectiva de los estudiantes de la Unidad Educativa Álvaro García Linera, Distrito Educativo Vinto.

No existe una relación directa entre la percepción liderazgo del director y la dimensión responsabilidad desde la perspectiva de los estudiantes del 3er año de escolaridad de la Unidad Educativa Álvaro García Linera.

\section{REFERENCIAS}

Carrera, B. (2002), Tendencias de liderazgo en el docente venezolano de la I etapa de educación básica: Revista "Estudio de casos". Investigación y postgrado, vol.17, № 2, pp. 197-222

Chiavenato, I. (1989). Introducción a la Teoría General de la administración. México: Mc Graw - Hill Interamericana de México Chiroque, S. (2006). Evaluación del desempeño docente. Lima: Instituto de Pedagogía Popular

Thieme, C. (2005) Liderazgo y eficiencia en la educación primaria El caso de Chile Tesis doctoral. Universidad autónoma de Barcelona España. Recuperado de http://ddd.uab.cat/pub/tesis/2006/tdx0621106-000957/cptj1de1.pdf 